\title{
CARS Measurement of Rotational and Vibrational Temperatures of Nitrogen Molecules in Nonequilibrium Flow behind Hypervelocity Shock Wave up to $7 \mathrm{~km} / \mathrm{s}$
}

\author{
By Hima Bindu Venigalla ${ }^{1)}$, Shota NinNomi ${ }^{1)}$, Masashi Oguro ${ }^{1)}$, Masanori OtA ${ }^{2)}$ and Kazuo MaenO ${ }^{2)}$ \\ ${ }^{1)}$ Graduate Student, Graduate School of Engineering, Chiba University, Chiba, Japan \\ ${ }^{2)}$ Graduate School and Faculty of Engineering, Chiba University, Chiba, Japan
}

(Received June 27th, 2011)

\begin{abstract}
In the present research the characteristics of nonequilibrium flow are studied by applying CARS (Coherent Anti-Stokes Raman Spectroscopy) method to hypervelocity shock waves in low-density air. Furthermore, in order to get more detail information on the flow behind the hypervelocity shock wave and to inspect whether the disturbance is seen at the shock wave front during the CARS measurement, radiation images behind the shock waves are obtained simultaneously. Finally, the CARS signals are detected behind hypersonic shock waves with a shock velocity of up to $7 \mathrm{~km} / \mathrm{s}$. In addition, the radiation distributions are obtained at the same experiment. The vibrational and rotational temperatures are estimated by spectral fitting method. It is found that the rotational temperature exceeds the vibrational temperature when shock wave velocity is $5 \mathrm{~km} / \mathrm{s}$ or more, where both temperatures remains lower than translational temperature.
\end{abstract}

Key Words: CARS, Shock Wave, Radiative Non-Equilibrium Flow, Ro/Vibrational Temperature Diagnostics

\section{Nomenclature}

$\begin{array}{lll}F & : & \text { vibrational energy term } \\ G & : & \text { rotational energy term } \\ I & : \text { light intensity } \\ N & : \text { number of density } \\ T & : \text { temperature } \\ c & : \text { velocity of light } \\ k & : \text { wave vector } \\ l & : \text { interaction length } \\ n & : \text { refractive index } \\ \Delta & : \text { population difference } \\ \Gamma & : \text { Raman line width } \\ \omega & : \text { frequency } \\ \chi & : \text { nonlinear electric susceptibility } \\ c_{p} & : \text { specific heat } \\ h & : \text { heat transfer coefficient } \\ \rho & : \text { density } \\ D_{s f} & : \text { distance from shock wave front }\end{array}$

Subscripts

$\begin{array}{lll}0 & : \text { free space (in vacuum) } \\ 1 & : \text { YAG laser } \\ 2 & : \text { dye laser } \\ 3 & : \text { CARS signal } \\ (3) & : \text { third order } \\ \mathrm{nr} & : \text { free space } \\ \mathrm{j} & : \text { level } \\ \mathrm{v} & : \text { vibrational } \\ \mathrm{r} & : \text { rotational }\end{array}$

\section{Introduction}

The hypervelocity shock waves are generated in front of the reentry space planes while entering into atmosphere from space. When the reentry velocity ranges from $5 \mathrm{~km} / \mathrm{s}$ to over $10 \mathrm{~km} / \mathrm{s}$, radiative heating from the shocked air ahead of the vehicle plays an important role on the heat flux to the wall surface of the vehicle structure as well as convective heating. In order to clarify the radiative characteristics of the shock wave, experimental studies have been carried out during the last decades by many researchers using shock tube devices ${ }^{1-3)}$.

In our previous works, a CCD camera system has been introduced into spectroscopic radiation observation of hypervelocity shock waves in low-density air ${ }^{4,5)}$. The system consists of an imaging spectrograph, a streak camera, a gated image-intensified CCD camera and a personal computer. Some interesting features have been obtained independently for the $2 \mathrm{D}$ radiation and for the spectral radiation behind shock waves with over $10 \mathrm{~km} / \mathrm{s}$ velocity in air ${ }^{6,7)}$.

To evaluate rotational and vibrational temperatures of $\mathrm{N}_{2}$ molecule from the spectroscopic data, we used Boltzmann plot method, which was based on the second positive band of nitrogen molecules. However, Boltzmann plot data are based on some assumptions and gives large errors. The large errors are because Boltzmann plot method uses equilibrium range of molecular excitations. On the other hand, Coherent Anti-Stokes Raman Spectroscopy (CARS) has been developed for temperature and concentration measurement in relatively slow range of the flows of combustion ${ }^{8-11)}$. The CARS method is a nonlinear, four-wave optical mixing technique for combustion, fast flow and so on ${ }^{12-14)}$. The CARS method produces a strong and coherent signal and this signal could be detected even in strong background luminosity.

In this study the CARS method is behind the hypervelocity 
shock waves in low-density air as the temperature determination technique. The measurement system consists of a Nd:YAG laser, a dye laser, an imaging spectrograph, two gated image-intensified CCD cameras and so on. The rotational and vibrational temperatures of $\mathrm{N}_{2}$ are determined by spectral fitting method between the experimental spectroscopic profile data and theoretically calculated profile data. As a result, we have accumulated the spectral data obtained from behind the shock waves that velocities are ranging from Mach number 12 to 22 . Furthermore, in order to improve the reliability of hypervelocity flow and temperature data, the radiation distribution behind the shock wave is also measured simultaneously.

\section{CARS Method}

The theory of CARS method has been developed in molecular physics. Incident laser beams at frequencies $\omega_{1}$ (pump beam) and $\omega_{2}$ (Stokes beam) interact through the third order nonlinear electric susceptibility $\chi_{\text {CARS }}^{(3)}$ to produce a strong coherent radiation $\left(\omega_{3}\right)$ at $\omega_{3}=2 \omega_{1}-\omega_{2}$ as follows,

$I_{3}\left(\omega_{3}\right)=\frac{\omega_{3}^{2}}{n_{1}^{2} n_{2} n_{3} c^{4} \varepsilon_{0}^{2}}\left|\chi_{C A R S}^{(3)}\right|^{2} I_{1}^{2}\left(\omega_{1}\right) I_{2}\left(\omega_{2}\right) l^{2}\left(\frac{\sin \frac{\Delta k l}{2}}{\frac{\Delta k l}{2}}\right)^{2}$

$\Delta k=2 k_{1}-k_{2}-k_{3}$

where $n_{1}, n_{2}$ and $n_{3}$ are the refractive index at $\omega_{1}, \omega_{2}$ and $\omega_{3}$, respectively, $c$ is the velocity of light, $l$ is interaction length, $k_{1}$, $k_{2}$ and $k_{3}$ are the wave vectors of the pump, Stokes and CARS beams, respectively, and $\varepsilon_{0}$ is the permittivity of free space. The CARS signal is enhanced on the condition of phase matching, $\Delta k=0$. Then four vectors form phase-matching diagram (folded BOXCARS) shown in Fig. 1. The incident laser beams $\left(k_{1}\right)$ and a Stokes beam $\left(k_{2}\right)$, are aligned in order to satisfy vector relation. $\chi^{(3)}$ CARS is shown by

$$
\chi_{C A R S}^{(3)}=\sum_{j} K_{j} \frac{\Gamma_{j}}{2\left(\omega_{j}-\left(\omega_{1}-\omega_{2}\right)\right)_{j}-i \Gamma_{j}}+\chi_{n r}
$$

where the $j$ summation is over rotational and vibrational transitions in the vicinity of $\omega_{1}-\omega_{2}, \Gamma_{j}$ is the Raman line width (FWHM), $\chi_{\mathrm{nr}}$ is a background contribution due to electrons and remote resonance, and $\Delta \omega_{j}$ is shown to be related to the Raman cross-section by ${ }^{15)}$

$$
K_{j}=\left.\frac{2 n_{1} c^{4}}{n_{2} \mathrm{~h} \omega_{2}^{4}} N \Delta \frac{d \sigma}{d \Omega}\right|_{j} \Gamma_{j}^{-1}
$$

where $N$ is the number density of the Raman active molecule, $\Delta_{j}$ is the population difference between the upper and lower rotation and vibration states, and $\left.(d \sigma / d \Omega)\right|_{j}$ is the cross-section for spontaneous Raman scattering.

On the assumption that molecules have Boltzmann distributions based on the rotational $\left(T_{r}\right)$ and vibrational $\left(T_{v}\right)$ temperatures, $\Delta_{j}$ can be expressed as

$$
\begin{aligned}
\Delta_{j}=\frac{(2 J+1) g_{I}}{Q_{r} Q_{v}}\left[\exp \left(\frac{-F_{v, J} h c}{k T_{r}}\right) \exp \left(\frac{-G_{v} h c}{k T_{v}}\right)\right. \\
\left.-\exp \left(\frac{-F_{v+1, J} h c}{k T_{r}}\right) \exp \left(\frac{-G_{v+1} h c}{k T_{v}}\right)\right]
\end{aligned}
$$

where $F_{v, j}$ and $G_{j}$ are the rotational and vibrational energy terms, respectively, $g_{I}$ is the spin degeneracy, 6 for even- $J$ rotational levels and 3 for odd- $J$ levels in $\mathrm{N}_{2}$, and $Q_{r}$ and $Q_{v}$ are the rotational and vibrational partition functions, respectively. From Eq. (1), (3) and (4), CARS signal is approximately proportional to the square of number density of molecule. Therefore, it is not easy to detect the CARS signal from low-pressure and radiating fast gas.

As written in the following section, the measured CARS spectroscopic profiles are fitted with calculated CARS spectra by treating $T_{v}, T_{r}, \Gamma$ and $\chi_{n r}$ as free parameters to decide these temperatures.

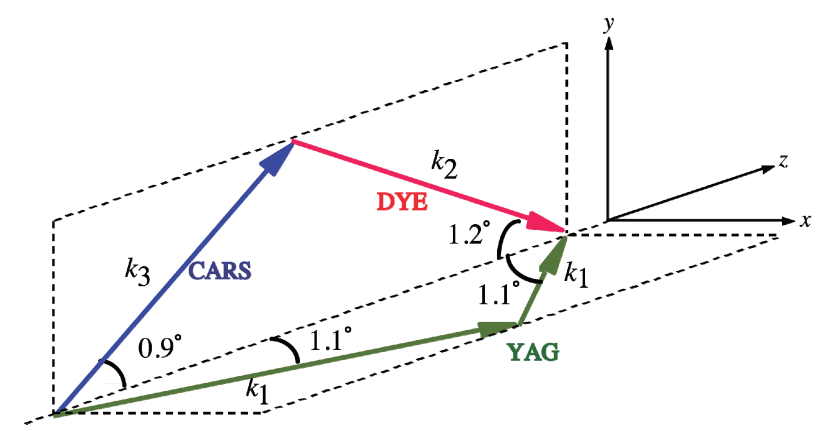

Fig. 1. Phase-matching diagram of folded BOXCARS.

Two pumping beams and a Stokes beam are focused into shock tube through a focal lens. The laser incidence vectors are shown in Fig. 1. The plane containing both $\omega_{2}$ and $\omega_{3}$ beams is perpendicular to the plane in two $\omega_{1}$ beams. In this case phase-matching condition has to be satisfied as well as planer BOXCARS. This method is called as folded BOXCARS, especially, multiplex folded BOXCARS uses broadband Stokes beam at single shot. This method has advantage in separating CARS signal from pumping and Stokes beams.

\section{Experimental Apparatus}

In this research a free piston, double-diaphragm shock tube has been used to generate strong shock waves in low-density air as shown in Fig. 2. This shock tube consists of high-pressure tube, compression tube, buffer tube and low-pressure tube. The test section of the low-pressure tube, where the CARS laser beams are crossed, is $40 \mathrm{~mm} \times 40 \mathrm{~mm}$ square. The observation window of the test section is mounted near a focal lens with some distance from the sidewall of the two small holes along the optical path of laser beam. The shock velocity is measured by using a couple of ion probes mounted inside the wall of the test-section. The adiabatic compression of helium ruptures the first steel diaphragm and 
generates strong shock wave in buffer tube. Then in the test section, rupturing of second aluminum diaphragm generates the hypervelocity shock wave ${ }^{16)}$.

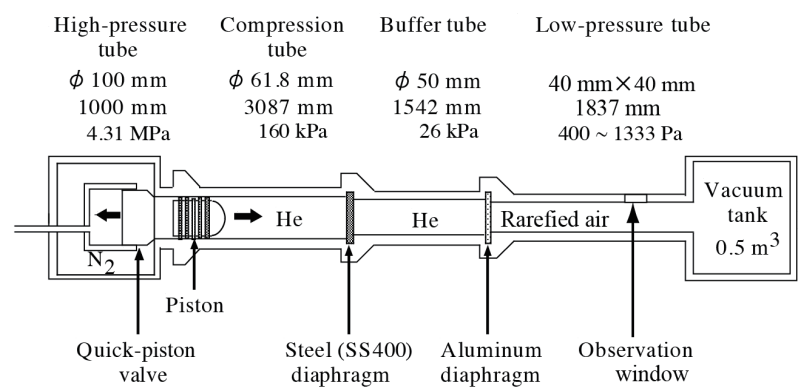

Fig. 2. Free-piston double-diaphragm shock tube.

Figure 3 shows a layout of diagnostic system for the CARS measurement in shock waves (CARS system). CARS system consists of a doubled Nd:YAG laser $\left(\omega_{1}\right)$, a dye laser $\left(\omega_{2}\right)$, a spectrograph, and an ICCD camera. The Nd:YAG laser (Continuum Precision 9010) is used for the pumping beam $\left(\omega_{1}\right)$. At a second harmonics beam $(532 \mathrm{~nm})$, the line width is $0.5 \mathrm{~cm}^{-1} \mathrm{FWHM}$, and the pulse duration is $10 \mathrm{~ns}$. The laser beam $\left(\omega_{1}\right)$ is divided in two lines by a beam splitter (BS). These beams are combined to the dye laser beam $\left(\omega_{2}\right)$ by a beam combiner (BC).

Then those beams are focused at measurement point of observation section. The dye laser is adjusted wavelength due to pigment of compounding. Rhodamine640 and Rhodamine610 are dissolved by methanol. The dye laser of Stokes beam is possible to oscillate at narrow band and broadband, in this study stokes beam is selected to be broadband. Due to the excitation by the YAG laser, Stokes beam delays a few ns. The optical path length of pump beam is adjusted to synchronize the arrival time of two beams at a measurement point. The CARS spectrum is detected by the ICCD. The entrance slit width is set to $100 \mu \mathrm{m}$ throughout the observation. The ICCD is mounted on the focal exit of the spectrograph.

The velocity of shock wave is obtained from the time difference in a couple of ion probes.

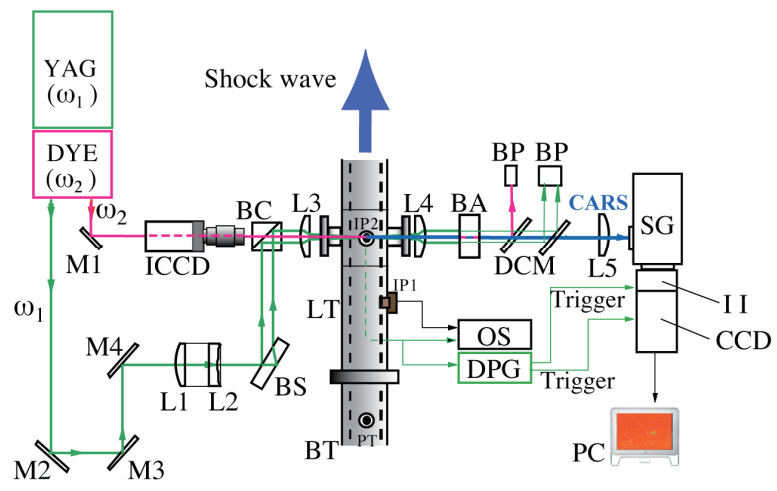

Fig. 3. Layout of spectroscopic system.

Figure 4 shows a section of observation part of the radiation distribution. The 2D radiation measurement system is constructed above the CARS system. The 2D radiation measurement system consists of a mirror (M5) and another CCD camera. CARS and 2D radiation measurement systems are structured so as not to disturb mutually, and then they enable us to obtain CARS signal and $2 \mathrm{D}$ radiation image simultaneously ${ }^{17}$.

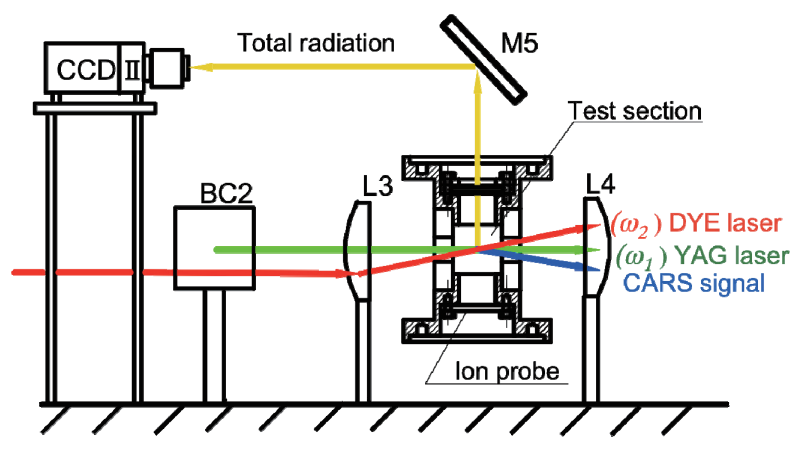

Fig. 4. Layout of 2D radiation measurement system.

In this study the synchronization of shock wave and laser beams to apply CARS method is strictly needed into the flow behind shock wave. According to the experimental data, shock wave velocity is well higher than $4 \mathrm{~km} / \mathrm{s}$. Measurement accuracy within $10 \mathrm{~ns}$ is necessary.

The three beams $\left(\omega_{1}\right.$ and $\left.\omega_{2}\right)$ must be irradiated on time when the running shock wave over $4 \mathrm{~km} / \mathrm{s}$ just arrives at the observation window. The trigger system synchronizing laser beams with shock wave is shown in Fig. 5. The Nd:YAG laser, which is an origin of three beams, is controlled by external signals. To begin with, a charge trigger is applied to charge a capacitance for flash lamp in YAG laser. Then flash lamp starts to flash by a fire trigger. After the fire trigger, the Q-switch trigger opens Q-switch, and starts the laser beam irradiation. Before the measurement, the charge and fire trigger is repeated. To generate shock wave, the circuit applies the signal to open the starting valve installed in a high-pressure tube, and simultaneously sends a charge trigger to the YAG laser. When shock wave arrives at a pressure transducer (PT) on the buffer tube, a delay pulse generator (DPG2) is triggered and sends a fire trigger signal with some delay. Finally, DPG1 is triggered by short-circuit of an ion probe with shock wave passing, and sends a Q-switch trigger to YAG laser. As shown in Fig. 5, DPG1 sends simultaneously triggers to the ICCD for detecting CARS signal. 


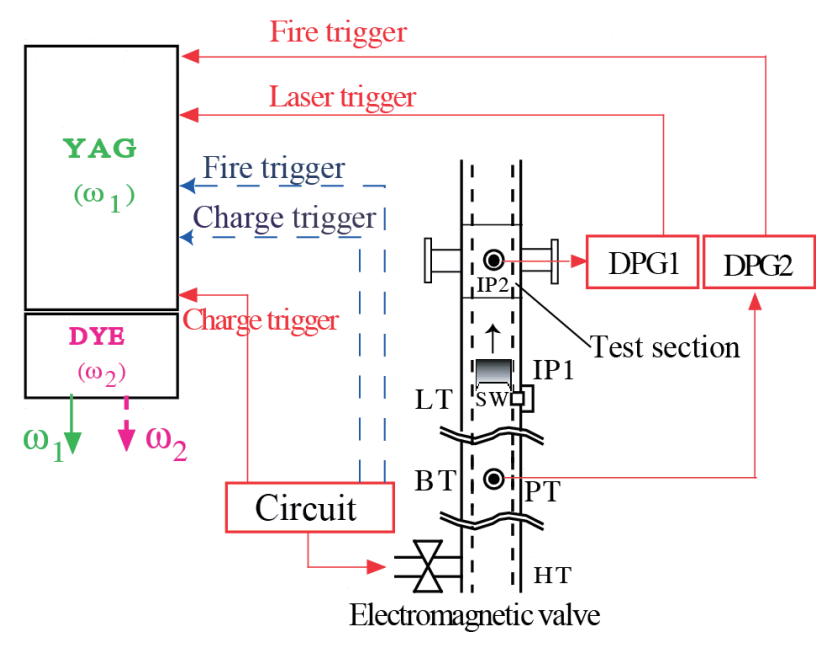

Fig. 5. Outline of the trigger system to synchronize laser beams with shock wave.

\section{Results and Discussion}

\subsection{Temperature measurement result (at shock velocity of $4 \mathrm{~km} / \mathrm{s}$ )}

Figure 6 and 7 show the typical experimental CARS signal obtained by spectrograph and spectral matching result. The flow direction in Fig. 6 cannot be determined because it is the spectroscopic signal obtained and it is irrespective of the flow direction and the pseudo color is obtained by the normalizing the intensity of the signal with the maximum intensity in the signal. The measurement point is $2.9 \mathrm{~mm}$ behind the shock wave front. Velocity of shock wave is $4.2 \mathrm{~km} / \mathrm{s}$ and Mach number is 12.4 . Initial pressure for the experiment is $2666 \mathrm{~Pa}$. As a result of spectral fitting method, the rotational and vibrational temperatures of nitrogen molecules are estimated $6000 \mathrm{~K}$ and $5000 \mathrm{~K}$, respectively. These values are obtained by the matching the experimental spectra with the spectra obtained theoretically using Eq. (1) to (5) and values where the minimum error is observed between theory and experimental spectra. It can be seen from the figure that experimental spectrum matches well with the theoretically calculated spectrum. Although both theoretical and experimental spectrum matches well, it can be observed that there are small discrepancies between both spectra; the reason may be because of the difficulty in calculating non-resonant terms theoretically in spectral fitting method. The error in both rotational and vibrational temperature measurement is of $\pm 500 \mathrm{~K}$.

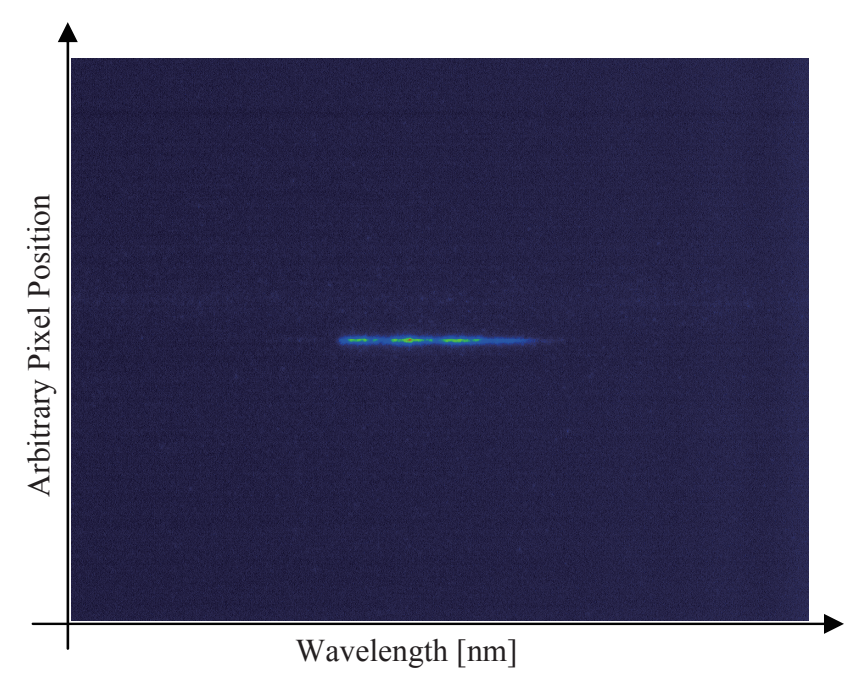

Fig. 6. Typical CARS signal obtained by spectrograph (pseudo color).

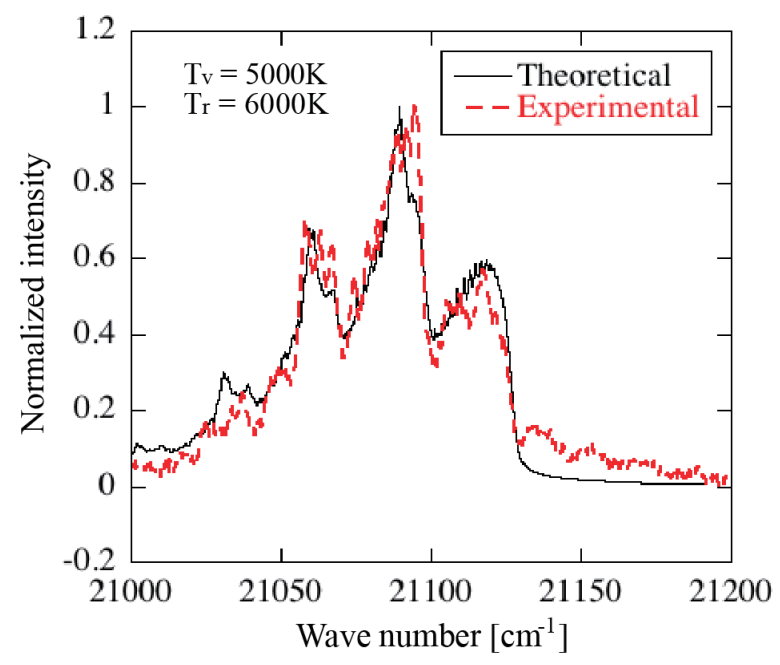

Fig. 7. Spectral fitting result at shock velocity of $4.2 \mathrm{~km} / \mathrm{s}$.

Figure 8 shows the rotational and vibrational temperatures distribution along $D_{s f}$ behind the shock wave front at shock velocity of around $4 \mathrm{~km} / \mathrm{s}$. In the right behind the shock wave front, the rotational and vibrational temperatures are distributed from $7500 \mathrm{~K}$ to $9500 \mathrm{~K}$, and $9000 \mathrm{~K}$, respectively. On the other hand, in the region behind $5 \mathrm{~mm}$ from shock wave front, the rotational and vibrational temperatures are appeared to distribute from $4000 \mathrm{~K}$ to $7000 \mathrm{~K}$. Since the differences in the rotational temperature and the vibrational temperature are not significant, there seems near equilibrium in rotational and vibrational energy behind the shock wave at velocity of about $4 \mathrm{~km} / \mathrm{s}$. Since Mach number 12.4 is the lower limit where the flow behind the shock wave front becomes radiative non-equilibrium and excitations between the energy levels in the nitrogen molecules are not very high which in turn shows the difference in temperatures behind the shock wave front are not varying significantly indicating that there exists near equilibrium flow behind the shock wave front. 


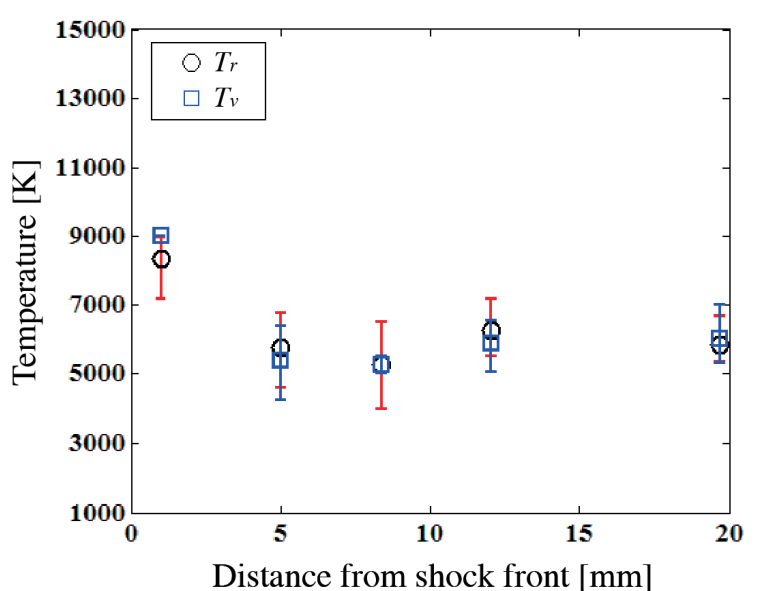

Fig. 8. Rotational and vibrational temperatures distribution along $D_{s f}$ at shock velocity of around $4 \mathrm{~km} / \mathrm{s}$.

\subsection{CARS-2D simultaneous measurement result (at shock velocity of $5 \mathrm{~km} / \mathrm{s} \sim 7 \mathrm{~km} / \mathrm{s}$ )}

We have performed detecting CARS signal and obtaining 2D radiation image simultaneously. The purpose of this experiment is to get more information on the flow behind the hypervelocity shock wave and to inspect whether the disturbance is seen at the shock front during the CARS measurement.

Figure 9 and 10 show the result of simultaneous measurements of CARS and 2D total radiation. The measurement position is $7.6 \mathrm{~mm}$ behind the shock wave front. Velocity of shock wave is $5.8 \mathrm{~km} / \mathrm{s}$ and Mach number is 17.1 . Initial pressure for the experiment is $1066 \mathrm{~Pa}$. In Fig. 9, shock wave is propagating from left to right in the round observation window. The dark portion in the Fig. 9 shows the flow ahead of the shock wave front and the brighter portion shows the flow behind the shock wave front indicating that there is radiative non-equilibrium flow. Figure 9 and 11 shows the normalized relative brightness to the maximum brightness of the figure. The darker region ahead of the shock front represents the stronger shock wave front and in turn less brightness in the total radiation figures. This is reason behind the difference in the brighter and darker regions of the Fig. 9 and Fig. 11. The rotational and vibrational temperatures are estimated $7500 \mathrm{~K}$ and $4500 \mathrm{~K}$, respectively. From the fitting result, rotational temperature considerably exceeds the vibrational temperature. Though experimental spectrum matches well with theoretically calculated spectrum, there remains a little discrepancy between theoretical spectrum and experimental spectrum in higher wave number region. This discrepancy is may be due to the difficulty in calculating the non-resonant part in theoretically calculated spectral fitting method. The error in both rotational and vibrational temperature measurements is $\pm 500 \mathrm{~K}$.

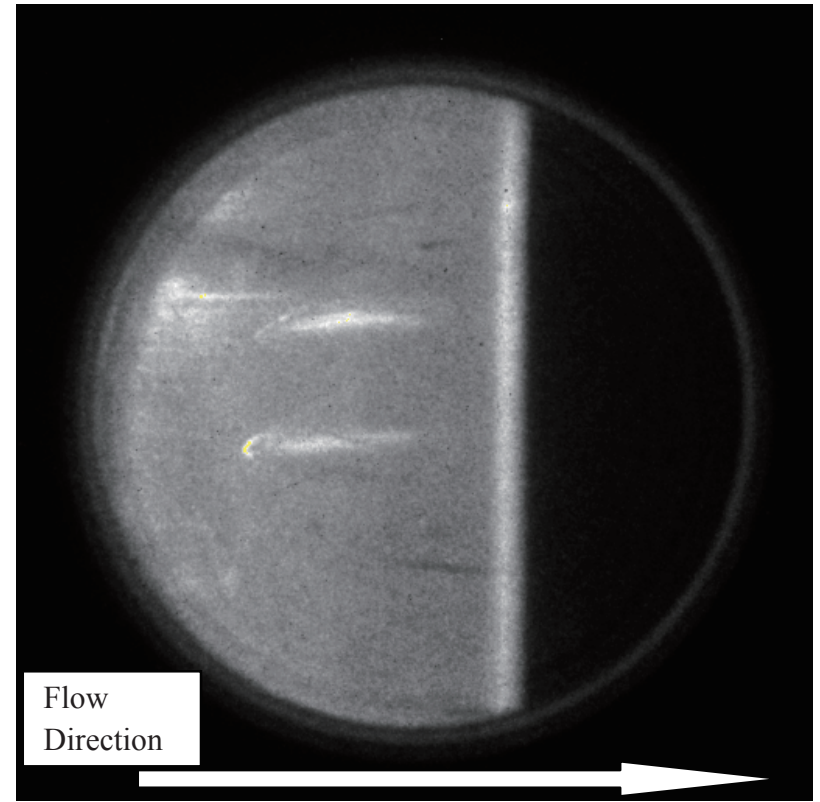

Fig. 9. 2D radiation image obatained at shock velocity of $5.8 \mathrm{~km} / \mathrm{s}$.

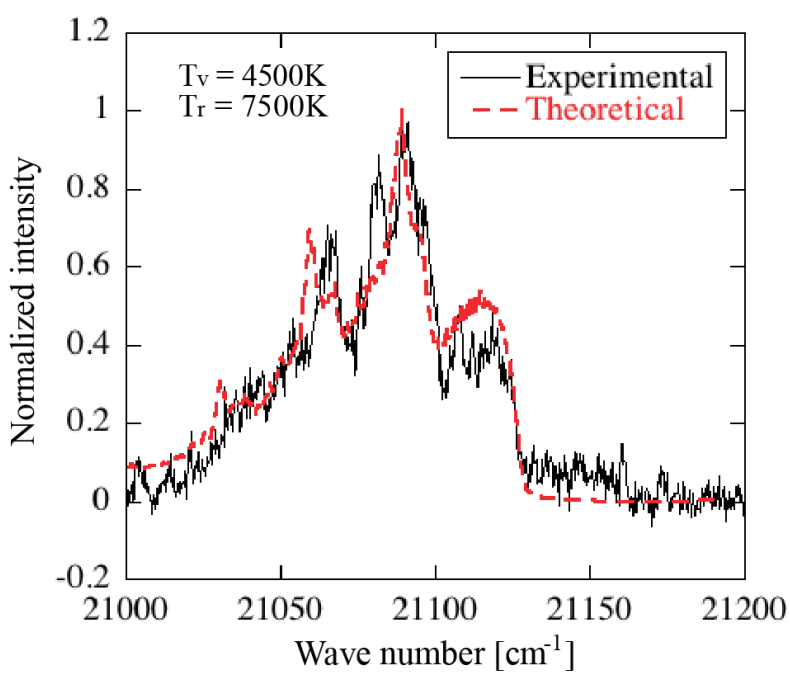

Fig. 10. Spectral fitting result at shock velocity of $5.8 \mathrm{~km} / \mathrm{s}$.

As the result of higher velocity of shock waves, the spectral fitting result and $2 \mathrm{D}$ radiation at shock velocity of $7.6 \mathrm{~km} / \mathrm{s}$ are shown in Fig. 11 and 12. The measurement position of CARS signal is $2.1 \mathrm{~mm}$ behind the shock front. Velocity of shock wave is $7.6 \mathrm{~km} / \mathrm{s}$, Mach number of 22.4. Initial pressure for the experiment is $400 \mathrm{~Pa}$. In Fig. 11, shock wave is propagating from left to right in the round observation window. As explained earlier, the darker portion and brighter portion in the Fig. 11 shows the flow ahead and behind the shock wave front respectively. The rotational and vibrational temperatures are estimated $6900 \mathrm{~K}$ and $2700 \mathrm{~K}$, respectively. Similarly, the rotational temperature considerably exceeds the vibrational temperature. However, due to the extensive disagreement is seen in the higher wave number region in the spectroscopic result, spectral fitting region is limited in lower wave number region which is unaffected by the non-resonant terms. This 
disagreement is most likely due to the lowering of $\mathrm{S} / \mathrm{N}$ ratio of CARS signal.

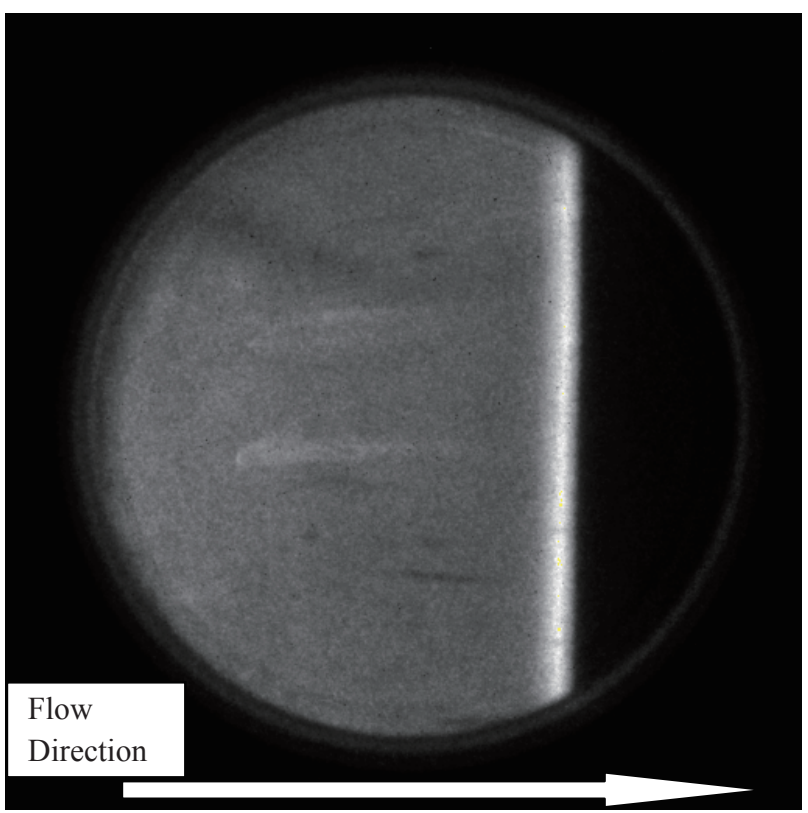

Fig. 11. 2D total radiation image obtained at shock velocity of $7.6 \mathrm{~km} / \mathrm{s}$.

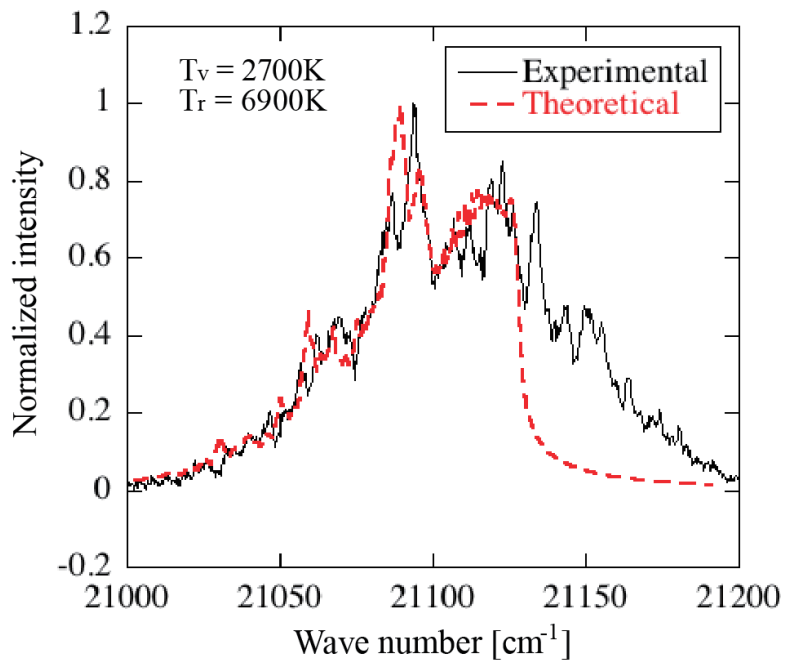

Fig. 12. Spectral fitting result at shock velocity of $7.6 \mathrm{~km} / \mathrm{s}$.

\section{Conclusion}

The rotational and vibrational temperatures behind the hypersonic shock wave have been satisfactorily measured by CARS method. Furthermore, the $2 \mathrm{D}$ radiation image is obtained simultaneously.

As a result, it would appear that a state of near equilibrium in rotational and vibrational energy seems to be measured at shock velocity of around $4 \mathrm{~km} / \mathrm{s}$, because great distinction between the rotational temperature and vibrational temperature cannot be seen in the present experiment. On the other hand, in over shock velocity of $5 \mathrm{~km} / \mathrm{s}$, the rotational temperature considerably exceeds the vibrational temperature in our results.

We need to obtain more CARS data to investigate the reliability of measured temperatures and clarify the hypervelocity shock-radiation nonequilibrium flows.

\section{References}

1) Honma, H. and Iizuka, H.: Experimental and numerical studies of radiations emission from high temperature air behind $10 \mathrm{~km} / \mathrm{s} \mathrm{shock}$ waves, In SAE Technical Paper Series, re-printed Internarional Pacific \& Space Technology Conference and 29th Aircraft Symposium Proceedings (1991).

2) Sharma, S. P. and Gillespie, W. D.: Non-equilibrium and equilibrium shock front radiation measurements, Journal of Thermophysics and Heat Transfer, 5(3) (1991), pp. 257-265.

3) Sharma, S. P.: Research on nonequilibrium phenomena at AMES e.a.s.t. facility, In Proceedings of 19th International Symposium on Shock Waves (1993), pp. 241-248.

4) Koreeda, J., Ohama, Y. and Honma, H.: Time resolved imaging spectroscopy of nonequilibrium shock-front radiation in air, In Proceedings of 20th International Symposium on Shock Waves (1995), pp. 1181-1186.

5) Koreeda, J., Ohama, Y. and Honma, H.: Imaging spectroscopy of the nonequilibrium shock front radaition in air, Shock Waves 8(2) (1998), pp. 71-78.

6) Honma, H., Maeno, K., Morioka, T., Matsuura, Y. and Koreeda, K.: Observation of radiation behind strong shock waves in air, Theoretical and Applied Mechanics, 45 (1996), pp. 231-239.

7) Morioka, T., Sakurai, N., Maeno, K. and Honma H.: Imaging spectroscopy of radiation behind strongshock waves in air, Theoretical and Applied Mechanics, 46 (1997), pp. 241-248.

8) Rado, W. G.: Nonlinear third order dielectric susceptibility coefficients of gases and optical third harmonic generation, Applied Physics Letters, 11(4) (1967), pp. 123-125.

9) Roh, W. B., Schreiber, P. W. and Taran, J. P. E.: Single-pulse coherent anti-Stokes Raman scattering, Applied Physics Letters, 29(3) (1976), pp. 174-176.

10) Eckbreth, A. C.: BOXCARS Crossed-beam phase-matched CARS generation in gases, Applied Physics Letters, 32(1) (1978), pp. $421-423$.

11) R'egnier, P. R. and Taran, P. E.: On the possibility of measureing gas concentrations by stimulated anti-Stokes scattering, Applied Physics Letters, 23(5) (1973), pp. 240-242.

12) Eckbreth, A. C., Dobbs , G. M., Stufflebeam, J. H. and Tellex, P. A.: CARS temperature and species measurements in augmented jet engine exhausts, Applied Optics, 23(9) (1984), pp. 1328-1339.

13) Boyce, R. R., Pulford, D. R. N., Houwing, A. F. P. and Mundt, Ch.: Rotational and vibrational temperature measurements using CARS in a hypervelocity shock layer flow and comparisons with CFD calculations, Shock Waves, 6 (1996), pp. 41-51.

14) Magre, P., Collin, G., Pin, O., Badie J., M., Olalde, G. and Cl'ement: Temperature measurements by CARS and intrusive probe in an air-hydrogen supersonic combustion, International Journal of Heat and Mass Transfer, 44 (2001), pp. 4095-4105.

15) Hall, R. J.: Cars spectra of combustion gases, Combustion and Flame, 35 (1979), pp. 47-60.

16) Honma, H., Tsukahara, M., Morioka, T., Katano, M. and Watanabe, N.: Characteristics of a free piston double-diaphragm shock tube (in Japanese), Journal of the Japan Society for Aeronautical and Space Science, 45(524) (1997), pp. 533-539.

17) Arimura, K., Miyazaki, K., Suhe, B., Ota M. and Maeno K.: Coherent anti-Stokes Raman spectroscopic measurement of radiation behind hypervelocity shock waves - CARS signal and radiation flow field, 19th ISSFD, 8 (2006), pp. 1-6. 